Tangence

De la révolution en fiction

Le banquier anarchiste de Fernando Pessoa

\title{
On the Revolution in Fiction
}

Fernando Pessoa's The Anarchist Banker

\section{Frédéric Rondeau}

Numéro 76, automne 2004

Figures de l'étrangeté. Proust, Musil, Pessoa, Cixous, Houellebecq

URI : https://id.erudit.org/iderudit/011216ar

DOI : https://doi.org/10.7202/011216ar

Aller au sommaire du numéro

\section{Éditeur(s)}

Tangence

ISSN

1189-4563 (imprimé)

1710-0305 (numérique)

Découvrir la revue

Citer cet article

Rondeau, F. (2004). De la révolution en fiction : Le banquier anarchiste de Fernando Pessoa. Tangence, (76), 51-67. https://doi.org/10.7202/011216ar
Résumé de l'article

En prenant pour objet Le banquier anarchiste, nouvelle où Fernando Pessoa s'interroge sur l'échec de la logique à expliquer la réalité, cet article propose de mettre en évidence les deux conceptions distinctes de l'anarchie qui y sont à l'oeuvre : la doctrine politique de l'anarchisme, mais aussi et surtout une forme d'“ anarchie esthétique ». Cette dernière, qui s'inspire de la première (ou serait-ce l'inverse ?), se signale par le questionnement de l'ordre établi et la remise en cause des conventions littéraires et sociales, mais surtout par une conception de l'individualité qui ne repose pas sur un rapport à l'(A)utre. Mise en parallèle avec certains essais d'Oscar Wilde et la théorie anarchiste de Max Stirner, cette lecture du Banquier cherche à tisser des liens étroits avec l'oeuvre entière de Pessoa. Le sentiment d'étrangeté au monde, qui procède notamment de la conscience de l'iniquité et de l'injustice, s'y présente paradoxalement comme étant, à la fois, source de la révolte et seul monde possible pour chacun. 


\title{
De la révolution en fiction: Le banquier anarchiste de Fernando Pessoa
}

\author{
Frédéric Rondeau, Université McGill
}

\begin{abstract}
En prenant pour objet Le banquier anarchiste, nouvelle où Fernando Pessoa s'interroge sur l'échec de la logique à expliquer la réalité, cet article propose de mettre en évidence les deux conceptions distinctes de l'anarchie qui y sont à l'œuvre: la doctrine politique de l'anarchisme, mais aussi et surtout une forme d'《anarchie esthétique ». Cette dernière, qui s'inspire de la première (ou serait-ce l'inverse?), se signale par le questionnement de l'ordre établi et la remise en cause des conventions littéraires et sociales, mais surtout par une conception de l'individualité qui ne repose pas sur un rapport à l'(A)utre. Mise en parallèle avec certains essais d'Oscar Wilde et la théorie anarchiste de Max Stirner, cette lecture du Banquier cherche à tisser des liens étroits avec l'œuvre entière de Pessoa. Le sentiment d'étrangeté au monde, qui procède notamment de la conscience de l'iniquité et de l'injustice, s'y présente paradoxalement comme étant, à la fois, source de la révolte et seul monde possible pour chacun.
\end{abstract}

Reconnaître dans la réalité une forme d'illusion, et dans l'illusion une forme de la réalité, est également nécessaire et également inutile.

Fernando Pessoa, Le livre de l'intranquillité

Chaque fois que les choses passent ou se passent bien, chaque fois qu'on applique tranquillement une bonne règle à un cas particulier, à un exemple correctement subsumé, selon un jugement déterminant, le droit y trouve peut-être et parfois son compte mais on peut être sûr que la justice n'y trouve jamais le sien.

Jacques Derrida, Force de loi 
«Notre vie n'avait pas de dedans. Nous étions dehors et autres. Nous ignorions tout de nous, comme si nous étions apparus à nos âmes au terme d'un voyage à travers des songes ${ }^{1} \ldots$... : cette remarque de Fernando Pessoa, plus que tout autre sans doute, fait immédiatement songer à ce "grand fantôme, [cette] inquiétante présence $^{2} »$ qui, depuis le romantisme, s'est répandu dans la littérature occidentale. De fait, Pessoa sera sans cesse hanté par cette inquiétante présence et écrira, en écho à la célèbre formule rimbaldienne: "Vivre, c'est être un autre ${ }^{3}$.» Il s'appliquera toutefois à conjuguer celle-ci au pluriel, faisant naître une multitude d'hétéronymes - dont Alberto Caeiro, Alvaro de Campos, Bernardo Soares - qui signeront à la fois poèmes et proses, que l'on pense à Ultimatum, au Gardeur de troupeaux ou encore au Livre de l'intranquillité. Pierre Léglise-Costa écrit: «L'hétéronyme Raphael Baldaya, ou Pessoa masqué, laisse présupposer qu'à un moment donné le poète a voulu créer ses créatures au point de déterminer les vies chronologiques des hétéronymes par une habile détermination mathématique partant de sa propre carte du ciel; d'où les variantes obligées, mais aussi l'unité reconquise, astrologiquement ${ }^{4}$.» De cette topologie déployée autour du nom Pessoa - «personne» en portugais, mais aussi persona, "masque» en latin - se constituent une généalogie fictive ainsi qu'une œuvre puissante et polymorphe ${ }^{5}$.

Étrangeté, quel autre terme pourrait mieux qualifier l'expérience de la distance par rapport au réel à laquelle nous invite Pessoa? L'auteur lui-même paraît vivre en retrait, dans une sorte d'absence au réel. Les photographies le représentent fuyant, spectral, revêtu d'un costume anonyme de commis ou de banquier. Il écrit d'ailleurs, au verso d'un portrait remis à sa tante, qu'il s'agit d'une «provisoire représentation visible de lui-même ${ }^{6}$ ». Un même

1. Fernando Pessoa, «Dans la forêt de l'absence», Proses publiées du vivant de l'auteur, traduction par Simone Biberfeld, Dominique Touati et Joaquim Vital, Paris, Éditions de la Différence, 1988, p. 145.

2. Antonio Tabucchi, "La nostalgie du possible et la fiction de la vérité », La nostalgie, l'automobile et l'infini. Lecture de Pessoa, Paris, Seuil, 1998, p. 18.

3. Fernando Pessoa, Le livre de l'intranquillité [en deux volumes: 1988 et 1992], nouvelle édition revue, corrigée et augmentée de nombreux inédits, traduction par Françoise Laye, Paris, Christian Bourgois éditeur, 1999, p. 125.

4. Pierre Léglise-Costa, "L'auteur et ses représentations", Magazine littéraire, Paris, septembre 1991, n² 291, p. 32.

5. Teresa Rita Lopes a révélé que Fernando Pessoa se créa jusqu'à 72 hétéronymes. Voir Jacobo Machover, "Les 72 hétéronymes", Magazine littéraire, Paris, septembre 1991, n² 291, p. 33.

6. «Fernando Pessoa», Magazine littéraire, Paris, septembre 1991, n² 291, p. 15. 
rapport trouble au réel se retrouve dans les écrits de Bernardo Soares, "semi-hétéronyme» de Pessoa, qui décrit, en ces termes, son inadéquation au monde:

Dans toutes les circonstances de ma vie [...] j'ai toujours été, aux yeux de tout le monde, un intrus. À tout le moins un étranger. Qu'il s'agisse de mes parents ou de mes amis, j'ai toujours été perçu comme quelqu'un du dehors [...] Pour ceuxlà mêmes qui, par nature, m'étaient les plus proches, j'ai toujours été une sorte d'invité et, en tant que tel, toujours bien traité, mais avec ce soin attentif que l'on doit aux étrangers, et ce manque d'affection auquel doit s'attendre tout intrus ${ }^{7}$.

Ce texte daté de 1917 se réfléchit dans une nouvelle de 1922, Le banquier anarchiste. Déjà, le titre en forme d'oxymore crée un effet performatif d'étrangeté. L'une des rares œuvres publiées du vivant de l'auteur — et signée de son «orthonyme» — parait dans le premier numéro de la revue Contemporânea. Robert Bréchon, dans une description enthousiaste, affirme qu'il s'agit là du "plus original des traités sur la liberté ${ }^{8}$ ». C'est d'ailleurs avec ce texte, stylistiquement éloigné du reste de son ouvre, que Pessoa souhaitait faire son entrée sur la scène littéraire européenne. L'année de sa mort (1935), dans une lettre adressée à Adolfo Casais Monteiro, il annonce la réécriture du Banquier anarchiste, ainsi que sa traduction en anglais en vue d'une éventuelle publication en Angleterre ${ }^{9}$. Ce désir de publier à l'étranger et la possibilité — selon Pessoa — d'y trouver le succès, tiennent au genre même de ce texte, qu'il qualifie de «conte de raisonnement» et qu'il situe dans la lignée d'Edgar Allan Poe.

La critique a cependant négligé Le banquier anarchiste. Si Bréchon considère qu'il s'agit d'un «de ces chefs-d'œuvre de Pessoa qui restent à redécouvrir ${ }^{10}$ ", les autres commentateurs semblent davantage n'y trouver qu'un stimulant exercice de style. Ellen Sapega constate également à quel point les jugements divergent chez ceux qui ont cherché à interpréter cette nouvelle, certains la dissociant des opinions politiques de Pessoa, d'autres affirmant au contraire que les tendances fascistes de l'auteur s'y

7. Robert Bréchon, Étrange étranger. Une biographie de Fernando Pessoa, Paris, Christian Bourgois éditeur, 1996, p. 348.

8. Robert Bréchon, Étrange étranger, ouvr. cité, p. 367.

9. On peut d'ailleurs lire quelques pages de cette traduction inachevée dans Fernando Pessoa, Le banquier anarchiste, traduction par Françoise Laye, Paris, Christian Bourgois éditeur, 2000.

10. Robert Bréchon, Étrange étranger, ouvr. cité, p. 366. 
donnent à lire ${ }^{11}$. Pessoa répond pourtant lui-même à ces accusations dans un texte où il souligne la distance que le lecteur doit adopter par rapport aux opinions exprimées dans ses textes:

On trouve, dans ces dédoublements de la personnalité, ou plutôt dans l'invention de ces personnalités différentes, deux degrés, ou types, qu'un lecteur attentif reconnaîtra à leurs caractères distinctifs. Au degré inférieur, la personnalité est marquée par des idées et des sentiments qui lui sont propres et tout à fait différents des miens; de même, au niveau le plus bas de ce premier degré, elle se distingue par des idées exposées au fil des raisonnements ou des arguments, mais qui ne sont pas les miennes, ou, si elles le sont, que j'ignore complètement. $L e$ banquier anarchiste est un exemple de ce niveau inférieur ${ }^{12}$.

Curieusement, alors que le personnage du banquier poursuit une réflexion sur l'anarchisme, dont il cherche à surmonter les faiblesses théoriques et à vaincre les apories, cet aspect de la nouvelle n'a pas été interrogé par la critique. L'anarchisme suscite l'inconfort, provoque un malaise. C'est d'ailleurs peut-être l'une des qualités de cette doctrine que Pessoa pouvait entrevoir. Tout anarchiste est essentiellement autre. En le rejetant dans les limbes de l'utopie, son intransigeance en fait un perpétuel étranger.

\section{Précis de l'anarchisme: lois, impasse et fiction}

La nouvelle de Pessoa s'ouvre sur un malentendu. Deux hommes terminent un repas: le narrateur et son "ami le banquier, commerçant et accapareur notoire ${ }^{13}{ }^{»}$. Le narrateur, cherchant à réanimer une conversation qui «était allée en mourant et gisait, maintenant morte», se souvient:

— Au fait: on me disait l'autre jour qu'autrefois, vous aviez été anarchiste...

- Que j'ai été, non: je l'ai été et je le suis toujours. Je n'ai pas changé sur ce point. Je suis anarchiste (BA, p. 11).

11. Ellen Sapega, "On Logical Contradictions and Contradictory Logic: Fernando Pessoa's O banqueiro anarquista", Luso - Brazilian Review, Madison (Wisconsin, É.-U.), vol. 26, nº 1, 1989, p. 111-118.

12. Fernando Pessoa, "De la préface aux Fictions de l'interlude», Le livre de l'intranquillité, ouvr. cité, p. 559.

13. Fernando Pessoa, Le banquier anarchiste, traduction par Françoise Laye, Paris, Christian Bourgois éditeur, 2000, p. 11. Désormais, les références à cet ouvrage seront indiquées par le sigle $B A$, suivi de la page, et placées entre parenthèses dans le corps du texte. 
Procédant par questions et réponses, le texte évoque la maïeutique socratique. Le personnage principal s'efforcera d'expliquer au narrateur que c'est précisément grâce à son métier de banquier qu'il est parvenu au véritable anarchisme. Hostile au préjugé tenace associant la doctrine révolutionnaire au chaos et au désordre, le banquier y voit plutôt, à la manière d'Élisée Reclus ${ }^{14}$, «la plus haute expression de l'ordre ${ }^{15} »$. À propos de certains anarchistes, "ces types [...] avec leurs bombes et leurs syndicats", le banquier pose d'emblée une distinction: «la différence entre nous, c'est qu'ils ne sont, eux, anarchistes qu'en théorie; moi, je le suis en théorie et en pratique» $(B A, \mathrm{p} .14)$.

Sa venue à l'anarchisme, nous explique-t-il, est issue du désir de comprendre sa rébellion: "[d]ès que je le pouvais, je lisais, je discutais et, comme je n'étais pas bête, il m'est venu une profonde insatisfaction, une révolte profonde contre mon sort et les conditions sociales qui l'imposaient» $(B A$, p. 16$)$. Cette colère s'exprime envers une multitude de forces qui, coalisées, condamnent la classe ouvrière à se perpétuer en la privant de doter ses descendants d'un héritage susceptible de les qualifier: "ni condition sociale, ni moyens de s'élever». Ce sont ses «dons naturels», "une intelligence lucide et une force de volonté assez marquée»( $B A, \mathrm{p} .15)$, qui constituent les armes du jeune ouvrier lisbonnais devenu anarchiste. Or, dans la nouvelle, le passage de l'état de révolté à celui de révolutionnaire - qui cherche à " comprendre cette révolte» - semble s'opérer naturellement. Pourtant, nous verrons comment le sentiment d'étrangeté au monde, qui est à la source de l'indignation, est subsumé dans une pratique anarchiste, puis intégré dans un combat révolutionnaire qui se donne précisément comme mission (peut-être insensée) d'abolir cette distance. Peutêtre s'agit-il là de la première aporie du discours du banquier anarchiste: en effet, est-il jamais possible de combattre l'étrangeté du dehors par le repli sur l'ipséité?

Interpellé par le narrateur qui lui demande pourquoi avoir choisi l'anarchisme — «cette solution extrême» (BA, p. 19) —

14. La pensée d'Élisée Reclus (1830-1905) se rapproche du communisme anarchiste de Kropotkine et de l'esprit mutualiste de Michel Bakounine. Emprisonné pour avoir participé à la Commune de Paris, il se voit par la suite contraint à l'exil. Il formera (avec Kropotkine et Bakounine) la Fédération jurassienne et organisera plusieurs réunions de propagande sur le territoire suisse. En tant que géographe, il écrit, en ces années, son œuvre maîtresse: Nouvelle géographie universelle.

15. Jean Préposiet, Histoire de l'anarchisme, Paris, Tallandier, 2002, p. 83. 
alors qu'il aurait pu opter pour une position intermédiaire, le socialisme par exemple, ou tout autre mouvement progressiste, le banquier répond:

Le grand mal, le seul à vrai dire, ce sont les conventions et les fictions sociales, qui se plaquent sur les réalités naturelles - oui, toutes les fictions, depuis la famille jusqu'à l'argent, depuis la religion jusqu'à l'État. On naît homme ou femme - je veux dire qu'on naît pour devenir, une fois adulte, homme ou femme; mais on ne naît pas, en bonne justice naturelle, pour être un époux, ou pour être riche ou pauvre, et pas davantage pour être catholique ou protestant, anglais ou portugais. On devient ceci ou cela en vertu des fictions sociales. Et ces fictions sociales, pourquoi sont-elles mauvaises? Parce que ce sont des fictions, parce qu'elles ne sont pas naturelles (BA, p. 20 ; c'est Pessoa qui souligne).

Dans ce raisonnement tautologique, la véritable justice est celle des "réalités naturelles», précédant les fictions de l'homme, dépouillées de l'État, de la religion, de l'argent... Mais, pour le banquier, la société "naturelle» ne saurait être accomplie réellement que si elle l'est totalement. Fidèle à son désir apparent de logique et de clarté, le banquier ajoute:

Donc, de deux choses l'une: ou le naturel est réalisable sur le plan social, ou il ne l'est pas; en d'autres termes, ou bien la société peut être naturelle, ou bien elle est essentiellement fiction et ne peut en aucun cas être naturelle. [...] [S]i [...] la société ne peut exister à l'état naturel, et si, pour une raison quelconque, elle doit absolument reposer sur des fictions, alors de deux maux choisissons le moindre; rendons-la, dans le cadre de ces fictions inévitables, la plus naturelle possible, afin de la rendre, par cela même, la plus juste possible (BA, p. 22).

À la suite de ce constat, le narrateur, tout comme le lecteur, croit avoir saisi le fonctionnement de l'anarchisme de pacotille du banquier: la société naturelle sera celle à laquelle nous sommes habitués, soit le capitalisme. Refusant toutefois l'hypothèse qu'une telle société ne puisse exister (pour le banquier, «ce qui est naturel l'est entièrement, et non à moitié, au quart ou au huitième» $(B A$, p. 21-22)), il rappelle au narrateur sa fidélité à la théorie anarchiste et refuse ainsi d'accepter l'idée d'une justice uniquement fondée sur des apparences naturelles: «Non: je devais faire quelque chose. Il fallait travailler, lutter pour la cause des opprimés, ces victimes des conventions sociales! [...] [Les hommes] peuvent tous être égaux entre eux; ce qui les en empêche, ce sont les fictions sociales, et c'est donc elles qu'il faut détruire» (BA, p. 31-32). 
Pour le banquier, c'est la «longue accoutumance des êtres humains aux fictions sociales, [créant] par elles-mêmes de la tyrannie» (BA, p. 39-40), qui est responsable de cette confusion. Une fois les conventions détruites, le banquier prévoit le retour de cette liberté qui serait le propre de l'homme. Cette idée d'un âge d'or - où la véritable liberté, désormais oubliée, était intacte constitue l'horizon anarchiste. Ernst Jünger écrit d'ailleurs à ce propos: "L'anarchiste, sous sa forme pure, est celui dont la mémoire remonte le plus loin, jusqu'à des ères préhistoriques, voire prémythiques - et qui croit que l'homme a rempli dans ces temps lointains sa vocation authentique ${ }^{16}$.»

\section{Technique de l'anarchisme}

Poursuivant la conversation, le banquier fait part au narrateur de la conscience qui est sienne d'une nouvelle emprise des fictions sociales sur ses anciens camarades anarchistes. À bien observer les activités du groupuscule révolutionnaire, il constate la création d'une forme de tyrannie encore plus perverse. Certaines personnes possèdent l'art, selon le banquier, de s'élever parmi les autres et de les entraîner à faire ce qu'elles souhaitent: "Un petit groupe de gens sincères, formé et uni expressément dans le but de travailler pour la liberté, n'avait réussi, au bout de plusieurs mois, qu'à créer une seule chose concrète et positive: de la tyrannie au sein même de ce groupe»( $B A$, p. 44 ; c'est Pessoa qui souligne). Le banquier ajoute que, si l'oppression était issue de cette longue exposition de l'humanité au mensonge, le problème aurait été détestable, mais dérivé d'un principe naturel. Il s'agissait toutefois d'une nouvelle forme de despotisme, "dénaturé», puisqu'il se créait au sein même de la lutte contre les fictions sociales. Si rien ne pouvait parvenir à résoudre cette difficulté, la découverte de "ce caractère terrible de toutes les tyrannies issues de la Nature: l'impossibilité de se révolter contre elles» $(B A$, p. 48$)$ mettrait fin au combat anarchiste. Le banquier affirme: «Et puis, un beau jour, d'un seul coup, j'ai trouvé la solution. Ce jour-là a été le grand jour de mes théories anarchistes: le jour où $j$ 'ai découvert, pour ainsi dire, la technique de l'anarchisme» (BA, p. 47).

L'oppression se développant à l'intérieur même du groupe, le banquier renoue avec l'individualisme, qui constitue le fondement

16. Ernst Jünger, L'État universel, suivi de La mobilisation totale, trad. de l'allemand par H. Plard et M. B. de Launay, Paris, Gallimard, 1990, p. 85. 
premier de l'anarchisme, et conclut que tous ceux qui s'en réclament devraient «tous travailler dans le même but, certes, mais... séparément» (BA, p. 51 ; c'est Pessoa qui souligne). Mal reçu par ses compagnons - puisque cette découverte doit s'accompagner de la dissolution du groupe - , il entreprend seul de suivre cette voie. Étranger absolu de l'anarchisme, en marge de la marge, le choix du banquier paraît correspondre à un "individualisme absolu ${ }^{17}$ » à la manière de Stirner. En effet, en cherchant à prolonger à l'extrême l'anarchisme, le banquier se rapproche de la pensée de Max Stirner qui, si elle en représente la forme sans compromis, correspond aussi à l'expression la plus idéalisée de la doctrine. La réflexion sur l'anarchie proposée par ce dernier repose sur l'expérience subjective du monde. Comme pour le banquier, ce qui intéresse le théoricien de l'anarchisme, ce n'est pas une quelconque «subjectivité transcendantale», mais bien sa propre présence, «son existence concrète et originale ${ }^{18} »$, ce qu'il nomme, paradoxalement, «l'Unique». Comme nous le verrons aussi chez Pessoa, il ne s'agit pas d'un déplacement du sacré de Dieu vers l'Homme, mais plutôt de l'abolition de la servitude au nom d'une cause supérieure et abstraite. Toutefois, l'anarchisme du banquier, bien qu'il témoigne de certaines similitudes avec elle, n'est pas calqué sur la pensée de Stirner. Une différence fondamentale se profile d'ailleurs dans le sort distinct que chacun réserve à la dimension collective de la lutte. Chez le banquier, si sa "technique de l'anarchisme» vise d'abord à se libérer soi-même, ce dernier s'intéresse aussi à la «libération» de tous. Alors que, "la véritable aliénation, la seule qui pose un problème à Stirner, c'est la sienne propre, en tant que sujet existant, en tant que monade. Ce n'est jamais celle du collectif humain ${ }^{19} »$. Marx reprochait ainsi à Stirner et à sa théorie de confondre le monde avec sa représentation - ce qui n'est pas sans rappeler l'approche du banquier.

Car, pour lui, la véritable et unique forme d'anarchisme consiste d'abord à se libérer soi-même pour ensuite lutter à la destruction des "fictions sociales". Puisqu'il considère l'argent comme la plus grande expression de la tyrannie, c'est d'emblée à cette emprise qu'il cherchera à échapper. En devenant banquier, en accumulant le plus d'argent possible (se libérant ainsi, semble-t-il, de son poids), il considère avoir atteint l'équilibre parfait entre

17. Jean Préposiet, Histoire de l'anarchisme, ouvr. cité, p. 121.

18. Jean Préposiet, Histoire de l'anarchisme, ouvr. cité, p. 128.

19. Jean Préposiet, Histoire de l'anarchisme, ouvr. cité, p. 130. 
théorie et praxis. Accusé de créer de la servitude autour de lui par l'exercice même de sa profession, le banquier déjoue à nouveau l'argument en prétextant qu'il n'y a pas, à proprement parler, création de tyrannie, celle-ci existant déjà. L'idée fondamentale consistant à ne pas y ajouter de cruauté arbitraire, le banquier observe qu'au surplus,

[la] tyrannie est le fait des fictions sociales, et non des hommes qui les incarnent: ceux-ci sont simplement, pour ainsi dire, les moyens que les fictions utilisent pour nous tyranniser [...] [D] étruisez donc tous les grands financiers détenteurs de capital dans le monde entier, mais sans détruire le capital. Dès le lendemain, le capital, passé en d'autres mains, continuera d'exercer sa tyrannie par le canal de ses nouveaux propriétaires. Maintenant, détruisez non pas les grands financiers, mais le capital luimême: combien de financiers restera-t-il? (BA, p. 68-69; c'est Pessoa qui souligne)

Évidemment, ce raisonnement suit une logique qui n'est pas à l'abri du sophisme. Qu'on en juge également d'après ce passage: "Comment me rendre maître de l'argent, tout en le combattant? Comment me dérober à son influence, à sa tyrannie, sans pour autant esquiver la rencontre? Il n'y avait qu'un moyen... EN GAGNER!»(BA, p. 62-63, Pessoa souligne). Difficile, en effet, de ne pas en conclure que, malgré sa théorie de la libération par l'acquisition, le banquier demeure toujours soumis au joug de l'argent.

La critique considère que la conclusion du Banquier anarchiste est absurde. Semblable à la nouvelle d'Oscar Wilde, The Soul of Man under Socialism - dans laquelle, partant du socialisme, la réflexion conduit à l'anarchisme - la nouvelle de Pessoa parcourt, en quelque sorte, le chemin qui mène de l'anarchisme jusqu'aux principes du capitalisme. Mais avant de considérer la fin de la nouvelle, examinons avec plus d'attention cette idée de "nature» ou de «naturel». Principe central qui légitime l'action du banquier, celle-ci se confond avec l'idée d'un paradis perdu pour l'homme et lui permet de poursuivre son projet sans avoir à se prononcer sur le fonctionnement éventuel d'une société postrévolutionnaire, tout développement à cet égard étant superflu, puisque l'homme, libéré des fictions sociales, regagnera alors ses instincts naturels. 


\section{Nature et justice}

Dans Force de loi, Jacques Derrida rappelait que «le droit est toujours une force autorisée ${ }^{20} »$. Or, le droit auquel en appelle le banquier dérive de l'autorité de la nature qui, en servant de socle à l'anarchisme, lui permet non seulement de ne pas s'envisager comme un autre type de fiction sociale, mais encore de refuser toute forme de transcendance. Négation du pouvoir absolu de Dieu et de l'hégémonie de l'Homme, l'anarchisme est une forme d'antihumanisme pour lequel les lois prescrites par la nature sont aussi celles du genre humain. L'on peut ajouter, en ce sens, que l'anarchisme cherche à (ré)instaurer l'hospitalité de la nature pour l'homme et que son projet, s'il se concrétisait, abolirait le sentiment d'étrangeté qui se trouve à la source même de la révolte du banquier.

Mais comment se fait-il que le personnage, au terme de son raisonnement, ne soit pas averti de l'incohérence de sa démarche, l'idéal de libération auquel il parvient ressemblant en tous points au capitalisme? Ellen Sapega note avec justesse que le banquier se crée sa propre «fiction individuelle» afin de contrer les «fictions sociales ${ }^{21} »$. En d'autres mots, la découverte que fait le protagoniste de la «parfaite» alliance entre théorie et pratique de l'anarchisme propose à chacun de créer son propre récit, de manière à ce que le discours et les actions de chaque individu ne répondent plus qu'aux exigences et aux devoirs qu'il se sera lui-même imposés.

C'est cependant autour de l'idée de nature que son raisonnement achoppe et devient invalide. On sait qu'en conclusion, le banquier revient sur ce qu'il prônait, c'est-à-dire une plus grande justice pour tous. Ce désaveu est, de fait, la conséquence nécessaire d'une idée de justice reposant sur l'obéissance à des lois qui sont en accord avec les règles que dicte la nature. Mais quelles sont ces règles? Ce sont celles que chacun, dans sa fiction individuelle, se fixe pour lui-même. Mais que devient alors l'idée même de règle? Jacques Derrida, dans Force de loi, citait ce passage célèbre de Montaigne: "Or les loix se maintiennent en credit, non parce qu'elles sont justes, mais parce qu'elles sont loix. C'est le fondement mystique de leur autorité, elles n'en ont poinct d'autre [...]. Quiconque leur obeyt parce qu'elles sont justes, ne leur obeyt pas

20. Jacques Derrida, Force de loi, Paris, Éditions Galilée, 1994, p. 17.

21. Ellen Sapega, "On Logical Contradictions and Contradictory Logic: Fernando Pessoa's O banqueiro anarquista», art. cité, p. 115. 
justement par où il doibt ${ }^{22}$ ». L'écueil sur lequel échoue le discours du banquier tient à l'impossibilité de marquer une différence entre loi et justice. Ce que souligne Derrida - et qui peut s'appliquer au lecteur de ce «conte de raisonnement»-, c'est que «[1]es lois ne sont pas justes en tant que lois. On ne leur obéit pas parce qu'elles sont justes mais parce qu'elles ont de l'autoritée ${ }^{23} »$. Derrida poursuit:

Montaigne proposait une analogie entre ce supplément de fiction légitime, c'est-à-dire nécessaire pour fonder la vérité de la justice et le supplément d'artifice appelé par une déficience de la nature, comme si l'absence de droit naturel appelait le supplément de droit historique ou positif, c'est-à-dire un surcroît de fiction, comme - et c'est le rapprochement proposé par Montaigne - «les femmes employent des dents d'yvoire où les leurs naturelles leur manquent, et, au lieu de leur vray teint, en forgent un de quelque matiere estrangere... s'embellissent d'une beauté fauce et empruntée: ainsi faict la science (et nostre droict mesme, a dict-on, des fictions légitimes sur lesquelles il fonde la vérité de sa justice) ${ }^{24}$.»

Pour le banquier, la nature correspond à cette «fiction légitime». Mais doit-on toujours considérer que la conclusion du banquier est «absurde»? Comment le raisonnement du personnage pourrait-il l'être, puisqu'il agit à l'intérieur de sa «fiction individuelle», dans cet espace quasi onirique où la théorie est légitimée par la pratique et inversement, sans jamais véritablement s'unir? Peut-il être à la fois un grand capitaliste et un grand anarchiste ${ }^{25}$ ? Pour un temps peut-être, puisqu'en accord avec son raisonnement, lorsque chacun sera parvenu à un pareil degré d'individualisme et libéré des fictions sociales, la révolution sera là, virtuellement, et n'attendra plus que sa concrétisation réelle. On retrouve alors à l'œuvre le même principe que dans Le déclin $d u$ mensonge d'Oscar Wilde: le principe général suivant lequel «la vie imite l'art bien plus que l'art n'imite la vie ${ }^{26} »$, les différentes idiosyncrasies investissant le réel pour le métamorphoser.

22. Montaigne, "De l'expérience», Essais III; cité par Jacques Derrida dans Force de loi, ouvr. cité, p. 29.

23. Jacques Derrida, Force de loi, ouvr. cité, p. 30.

24. Jacques Derrida, Force de loi, ouvr. cité, p. 30-31; c'est moi qui souligne.

25. Il n'est pas ici question de la doctrine de l'anarcho-capitalisme, qui provient entre autres de l'anarchisme individualiste de Max Stirner. Il faut se rappeler que le banquier de Pessoa vise l'abolition du capital.

26. Oscar Wilde, Le déclin du mensonge, Bruxelles, Éditions Complexe, coll. «Le regard littéraire», 1986, p. 65. 
Par la création de sa fiction individuelle, le banquier exprime un refus catégorique du monde bourgeois et parvient à se rapprocher comme jamais de l'idéal anarchiste. Cependant, s'agit-il ici du combat d'un révolté ou d'un révolutionnaire? On peut avoir l'impression que sa «technique de l'anarchisme» constitue un recul, un approfondissement du sentiment d'étrangeté où tend à se perdre la force de l'indignation qui l'animait à ses débuts. Il faut toutefois comprendre que, de son point de vue, il s'agit d'un véritable acte révolutionnaire, puisqu'il parvient au plus près de l'abolition du sentiment d'étrangeté qui l'animait devant le spectacle de l'injustice et de l'iniquité. C'est donc dire que, par sa fiction, une forme de communion entre lui-même et le monde s'est créée: tout, dans son récit, reflète la cohérence, la justice et l'égalité.

\section{«L'art moderne est l'art du rêve ${ }^{27}$ »}

C'est avec ce «conte de raisonnement» que Fernando Pessoa a cherché à faire son entrée dans le champ littéraire européen. Dans une série de textes écrits quelques années auparavant, Pessoa interrogeait les grands courants esthétiques européens afin d'identifier (autre tâche immense) la singularité de l'Europe. Pessoa y insistait notamment sur le fait qu'à son sens, le seul grand apport du romantisme tenait à la «spéculation et la réflexion", avant de préciser que ce courant «se rattache à la grande tradition de la civilisation européenne, c'est-à-dire à la tradition hellénique de l'individualisme rationaliste ${ }^{28}$ ». Tous ces éléments — la «spéculation», la «tradition hellénique», l' «individualisme rationaliste» — sont présents sous une forme exacerbée dans Le banquier anarchiste. Bien que son auteur ait exprimé peu de respect envers le «mauvais chrétien décadent ${ }^{29}$ » que semble être Oscar Wilde, la nouvelle de Pessoa est entièrement imprégnée de textes comme The Decay of Lying, The Critic as Artist et The Soul of Man under Socialism ${ }^{30}$. De

27. Fernando Pessoa, "L'art moderne, art du rêve", Le chemin du serpent, choix de textes traduits par Michel Chandeigne, Françoise Laye et Jean-François Viégas, Paris, Christian Bourgois éditeur, 1991, p. 103.

28. Fernando Pessoa, "Classicisme décadent et romantisme», Le chemin du serpent, ouvr. cité, p. 98.

29. Fernando Pessoa, "Antonio Botto et l'idéal esthétique au Portugal», Proses publiées du vivant de l'auteur, ouvr. cité, p. 249.

30. Suzette Macedo exprime le même point de vue: «But I am convinced that Pessoa was, in fact, directly influenced by Wilde, not the Wilde of Salome (though the sickly necrophiliac eroticism of Antinous has a distinctly 
fait, ce sont les mêmes tensions que celles qui sont à l'œuvre dans The Decay of Lying qui se rejouent dans Le banquier anarchiste, avec ses conflits ouverts entre la réalité et la fiction, le naturel et l'artificiel, l'unique et l'universel. De ce point de vue, Le banquier anarchiste peut même être considéré comme un pastiche de la nouvelle de Wilde: du moins pouvons-nous le lire comme sa version politique - mais une version politique qui le surpasserait par le caractère excessif de la réflexion.

On se souvient sans doute qu'à l'occasion de cette nouvelle, Oscar Wilde entendait offrir une réponse polémique aux auteurs réalistes. Le personnage de Vivian, en particulier, cherchait à y établir auprès de son interlocuteur, Cyril, l'idée suivant laquelle il s'agit d'une erreur pour l'artiste que de représenter le réel. Aux partisans de la mimesis, Vivian répondait en proposant d'écrire un plaidoyer en faveur du «mensonge dans l'art ${ }^{31} »$. Pour ce dernier, partout «où l'art est retourné à la nature et à la vie, il est devenu vulgaire, banal, sans intérêt ${ }^{32} »$. Au risque de donner à son propos un accent sophistique, Vivian soutient même que c'est l'art qui influence la vie et non l'inverse: "À qui donc, sinon aux impressionnistes, devons-nous ces admirables brouillards fauves qui se glissent dans nos rues, estompent les becs de gaz, et transforment les maisons en ombres monstrueuses ${ }^{33}$ ? » Jean Vogel, à ce propos, évoque la "subversion des procédés de la saine logique ${ }^{34}$ » dans $L e$ déclin du mensonge. Le banquier anarchiste semble, à cet égard, se situer dans le même esprit. Chez Wilde comme chez Pessoa, "[1]a vérité est purement et simplement question de style ${ }^{35}$ », et la forme idéale qui permet au style d'incarner cette prétention semble être celle du dialogue platonicien. Plus près du monologue que du véritable dialogue - l'un des personnages ne servant qu'à relancer la conversation - , cette forme avait fait l'objet de précédents essais chez Pessoa, notamment à propos de la tyrannie ${ }^{36}$. Comme

"Salomean" aura) but Wilde, the writer of discursive prose» («Fernando Pessoa's O Banqueiro Anarquista and The Soul of a Man under Socialism ", Portuguese Studies, Londres, $\mathrm{n}^{\circ}$ 7, 1991, p. 106).

31. Oscar Wilde, Le déclin du mensonge, ouvr. cité, p. 27.

32. Oscar Wilde, Le déclin du mensonge, ouvr. cité, p. 50.

33. Oscar Wilde, Le déclin du mensonge, ouvr. cité, p. 67.

34. Jean Vogel, "L'esthétique d'Oscar Wilde», dans Oscar Wilde, Le déclin du mensonge, ouvr. cité, p. 90.

35. Oscar Wilde, Le déclin du mensonge, ouvr. cité, p. 15.

36. On retrouve ces «Cinq dialogues sur la tyrannie» dans Le chemin du serpent, ouvr. cité. 
chez Wilde, ce dernier postule le pouvoir qu'a l'art de transformer la vie. Pessoa, cependant, pousse jusqu'à l'excès les conséquences de cette perspective, entraînant ainsi le lecteur dans le délire de cette "logique irréelle». C'est à propos du Banquier anarchiste qu'Ellen Sapega écrit:

Nous faisons face à l'inquiétante conclusion que la logique et la réalité, comme nous les concevons traditionnellement, sont incompatibles et même antagonistes. Cette double contrainte indique que nous devrions rejeter la méthode du banquier et tenter de trouver une façon plus "authentique», qui se situerait ailleurs que dans la logique, afin d'expliquer notre relation au monde ${ }^{37}$.

À ce titre, la nouvelle de Pessoa ne peut être considérée ni comme un traité politique, ni comme un traité d'esthétique. Il s'agit davantage, de façon analogue à l'anarchisme, d' "un esprit, [d'] une manière d'être au monde ${ }^{38}{ }^{\prime}$. Dans un texte de 1916, Pessoa met d'ailleurs à jour l'aporie, la double contrainte structurant son raisonnement:

Toute connaissance vient des sens ou par eux; mais nous ne savons pas combien il y a de sens. [...] La raison, ou l'intellect, ne perçoit ni ne crée; elle ne fait que comparer, et, par comparaison, rectifie et élabore les données que les sens fournissent. La raison est, par conséquent, incompétente pour déterminer une vérité, pour cette raison qu'elle ne peut pas déterminer un fait, mais seulement le comparer à d'autres. [...] Le fait qu'il y ait un problème ne suppose pas forcément qu'il y ait une solution à ce problème. [...] Il n'y a de solution satisfaisante pour aucun problème social ${ }^{39}$.

Pour Oscar Wilde, le «réel» vient puiser à l'art, alors que pour Pessoa, la fiction est le réel individuel, le destin subjectif. Il y a, selon lui, impossibilité même à se situer ailleurs que dans cette fiction. En lieu et place des fictions sociales, il faut donc compter sur une multitude de récits. Le banquier anarchiste témoigne de

37. «We are facing the disquieting conclusion that logic and reality, as we wish them to be, are incompatible and even antagonistic. This double bind indicates that we should reject the Banker's method and try to find a "truer", non-logical way of explaining our relation to the world» (Ellen Sapega, "On Logical Contradictions and Contradictory Logic: Fernando Pessoa's $O$ banqueiro anarquista", art. cité, p. 113). Je traduis.

38. Jean Préposiet, Histoire de l’anarchisme, ouvr. cité, p. 13; c'est Préposiet qui souligne.

39. Fernando Pessoa, «Les sens», Le chemin du serpent, ouvr. cité, p. 78-79. 
l'incapacité à suppléer aux lacunes de la vie, aux défauts de la réalité empirique (par un système plus juste), tout en soutenant la possibilité d'une perfectibilité par l'art.

\section{Une multitude de récits}

On trouve, dans un texte de 1912, une phrase de Pessoa qui peut prendre la forme d'un appel:

On assiste, à l'Âge moderne, à la scission entre la pensée et l'action, entre d'une part la pensée de l'effort et l'idéal, et d'autre part l'effort en lui-même et sa réalisation. [...] [De nos jours], [1]e moindre rêve est immédiatement accompagné par l'inévitable pensée de son impossibilité ${ }^{40}$.

Le banquier anarchiste serait-il une intimation à briser ce schème? Cette nouvelle ne constitue certes pas un programme politique. D'ailleurs, malgré le fait que Pessoa ait été un véritable caméléon politique, on ne peut retrouver, chez lui, aucune trace d'une quelconque proximité avec l'anarchisme ${ }^{41}$. Que signifie donc pour lui ce mot que l'on retrouve au hasard de ses textes, et ce, dès les premières années d'écriture? Tantôt manifestation du désordre et du chaos et tantôt geste salutaire et libérateur, l'anarchisme est une «doctrine» dont la présence dans l'œuvre semble surtout correspondre à celle du rêve, de l'étrangeté, de l'art comme « une forme de l'exagération ${ }^{42}$ ». Plus explicitement encore, un poème inédit de Pessoa, intitulé "Anarchisme» — dont le style est semblable à celui de son hétéronyme Alberto Caeiro (avec son mysticisme panthéiste et sa nostalgie que les Portugais nomment «saudade») —, permet de rapprocher Le banquier anarchiste de l'œuvre poétique:

[...]

Dans le fond je suis le même que Dieu.

Ma présence actuelle contient les âges antérieurs à la vie, les temps plus vieux que la terre, les trous de l'espace avant

40. Fernando Pessoa, «L'art moderne, art du rêve», Le chemin du serpent, ouvr. cité, p. 103.

41. À cette exception près où Pessoa lance un délirant appel: «Cultivons en nousmêmes, telle une fleur rare, la désintégration mentale. Construisons une anarchie portugaise » ("L'excès de discipline», Le chemin du serpent, ouvr. cité, p. 236).

42. Oscar Wilde, Le déclin du mensonge, ouvr. cité, p. 47. 
que le monde soit.

[...]

Shakespeare fait partie de moi. Cromwell a travaillé pour moi quand il a construit l'Angleterre. En triomphant de Rome, Henri VIII a fait de moi ce que je suis. Pour moi, Aristote a pensé et Homère chanté. En un sens mystique et profond véritablement $[\ldots]$, le Christ est mort pour moi. Il y a deux mille ans, un mystique indien dont j'ignore s'il a existé a pris part à mon être actuel. À ma présence d'aujourd'hui Confucius a édicté une morale. Le premier homme qui a découvert le feu, celui qui a inventé la roue, celui qui a conçu la flèche - si, aujourd'hui je suis moi c'est parce qu'ils ont existé ${ }^{43}$.

Où se trouve l'anarchisme dans ce poème si ce n'est, comme dans la nouvelle, dans la volonté d'abolir les conventions? Pour le poète, Jésus, Confucius, Aristote, ainsi que l'univers entier se côtoient et convergent vers lui. L'anarchisme, dans ce poème, incarne toute la potentialité de l'homme, dans son infinie grandeur, dans sa capacité à recevoir «les âges antérieurs à la vie» et dans son infinie absence: "Dans le fond je suis le même que Dieu».

Mise en parallèle avec celle des essais de Wilde et de la théorie anarchiste de Max Stirner, la lecture du Banquier anarchiste permet enfin, me semble-t-il, de tisser des liens étroits avec l'œuvre entière de Pessoa. Car s'il est vrai, comme le considèrent les critiques, que la forme empruntée par l'écrivain dans cette nouvelle demeure marginale dans son corpus, la présence de la "fiction individuelle» du banquier - et donc de la multitude de récits qu'il nous invite à imaginer - nous guident inévitablement vers l'essaim hétéronymique caractéristique de Fernando Pessoa. Ce qui importe chez Stirner, dans ce poème ou même pour le banquier, n'est pas tant de «changer l'ordre du monde, mais plutôt [d'en] modifier sa conscience $[. .$.$] par un renversement radical de l'attitude naturelle$ à l'égard de la réalité ${ }^{44}$ ». Chez Pessoa, la méfiance envers toute forme d'unité métaphysique trouve son assurance dans une

43. Fernando Pessoa, "Anarchisme», Magazine littéraire, Paris, septembre 1991, $\mathrm{n}^{\circ} 291$, p. 29.

44. Jean Préposiet, Histoire de l'anarchisme, ouvr. cité, p. 146-147. 
conception de l'individualité qui demeure au-delà de toute pratique et de toute logique, si bien que le rêve n'apparaît pas comme un double du monde, mais comme le seul monde possible pour chacun. 